\title{
A bifunctional aminoglycoside acetyltransferase/ phosphotransferase conferring tobramycin resistance provides an efficient selectable marker for plastid transformation
}

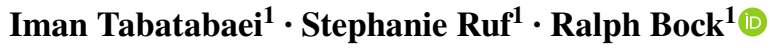

Received: 30 September 2016 / Accepted: 10 November 2016 / Published online: 17 November 2016

(C) The Author(s) 2016. This article is published with open access at Springerlink.com

\begin{abstract}
Key message A new selectable marker gene for stable transformation of the plastid genome was developed that is similarly efficient as the aadA, and produces no background of spontaneous resistance mutants.

Abstract More than 25 years after its development for Chlamydomonas and tobacco, the transformation of the chloroplast genome still represents a challenging technology that is available only in a handful of species. The vast majority of chloroplast transformation experiments conducted thus far have relied on a single selectable marker gene, the spectinomycin resistance gene aadA. Although a few alternative markers have been reported, the aadA has remained unrivalled in efficiency and is, therefore, nearly exclusively used. The development of new marker genes for plastid transformation is of crucial importance to all efforts towards extending the species range of the technology as well as to those applications in basic research, biotechnology and synthetic biology that involve the multistep engineering of plastid genomes. Here, we have tested a bifunctional resistance gene for its suitability as a selectable marker for chloroplast transformation. The bacterial enzyme aminoglycoside acetyltransferase(6')-Ie/aminoglycoside phosphotransferase(2")-Ia possesses an N-terminal acetyltransferase domain and a C-terminal phosphotransferase domain that can act synergistically and detoxify
\end{abstract}

Electronic supplementary material The online version of this article (doi:10.1007/s11103-016-0560-x) contains supplementary material, which is available to authorized users.

Ralph Bock

rbock@mpimp-golm.mpg.de

1 Max-Planck-Institut für Molekulare Pflanzenphysiologie, Am Mühlenberg 1, 14476 Potsdam-Golm, Germany aminoglycoside antibiotics highly efficiently. We report that, in combination with selection for resistance to the aminoglycoside tobramycin, the $\operatorname{aac}\left(6^{\prime}\right)$-Ielaph(2")-Ia gene represents an efficient marker for plastid transformation in that it produces similar numbers of transplastomic lines as the spectinomycin resistance gene aadA. Importantly, no spontaneous antibiotic resistance mutants appear under tobramycin selection.

Keywords Plastid transformation - Nicotiana tabacum . Selectable marker $\cdot$ Tobramycin $\cdot$ Bifunctional enzyme

\section{Introduction}

The development of technologies to engineer plastid (chloroplast) genomes (Boynton et al. 1988; Svab et al. 1990) has opened up new exciting opportunities to study virtually all aspects of plastid biology using in vivo approaches. Over the last three decades, application of the technology has provided novel insights into plastid gene expression (e.g., Kuras and Wollman 1994; Staub and Maliga 1993; Hajdukiewicz et al. 1997; Bock and Koop 1997), chloroplast gene functions (e.g., Monod et al. 1994; Ruf et al. 1997; Hager et al. 1999), plastid inheritance (Ruf et al. 2007; Svab and Maliga 2007) and genome evolution (Huang et al. 2003; Stegemann et al. 2003, 2012). Moreover, plastid transformation technologies have also stirred considerable excitement among plant biotechnologists, because transgene expression from the plastid genome offers a number of unique attractions, such as foreign protein expression to very high levels (De Cosa et al. 2001; Oey et al. 2009a), convenient stacking of multiple transgenes in synthetic operons ( $\mathrm{Lu}$ et al. 2013) and improved transgene containment due to the maternal mode of plastid inheritance 
in most crops which largely prevents unwanted transgene transmission via pollen (reviewed, e.g., in Maliga 2004; Bock 2015). In recent years, a large number of proof-ofconcept studies have demonstrated the great potential of the transplastomic technology in molecular farming (Staub et al. 2000; Tregoning et al. 2003; Oey et al. 2009b), metabolic engineering (Apel and Bock 2009; Bohmert-Tatarev et al. 2011; Fuentes et al. 2016) and resistance engineering (De Cosa et al. 2001; Ye et al. 2001; Zhang et al. 2015).

A major technical challenge still lies in the extension of the plastid transformation technology to new species and, especially, to major food crops (Bock 2014). Currently, plastid engineering is restricted to a handful of species, with the unicellular alga Chlamydomonas rheinhardtii and the seed plant model tobacco (Nicotiana tabacum) being the only species where the technology is routine in at least a number of laboratories. In addition to limitations related to the available tissue culture systems and plant regeneration protocols, the paucity of selectable marker genes for plastid transformation and the complete lack of suitable selection markers for certain groups of species represent the most serious obstacle to the development of workable transformation protocols for additional species. For example, cereals, the world's most important food crops, are recalcitrant to chloroplast transformation, because they are naturally resistant to spectinomycin (Fromm et al. 1987), the most commonly used antibiotic for the selection of transplastomic cells. The lack of a suitable selectable marker gene is also the main obstacle to the development of a mitochondrial transformation technology in plants ( $\mathrm{Li}$ et al. 2011).

The nearly universally employed selectable marker gene for plastid transformation is the aadA gene (GoldschmidtClermont 1991; Svab and Maliga 1993). It was identified in a strain of the gut bacterium Escherichia coli and encodes an aminoglycoside 3 "-adenylyltransferase. This enzyme covalently modifies the aminoglycoside antibiotics spectinomycin and streptomycin by attaching an AMP residue to the antibiotic molecules. Unlike the unmodified antibiotics, the adenylylated drugs do not bind to the $30 \mathrm{~S}$ subunit of the prokaryotic 70S ribosomes of the chloroplast and, therefore, do not block plastid protein biosynthesis. The identification of spectinomycin as selection agent for transplastomic cells in conjunction with the aadA marker (Goldschmidt-Clermont 1991; Svab and Maliga 1993) was a lucky strike. Despite great efforts to develop alternative markers, the aadA gene has remained unparalleled in its efficiency. This is likely due to the high enzymatic activity of the AadA protein and the high specificity of spectinomycin as a potent inhibitor of plastid translation. A few alternative selectable markers have been developed for tobacco plastid transformation, including the nptII gene encoding a neomycin phosphotransferase that confers resistance to kanamycin (Carrer et al. 1993), the aphA-6 gene that encodes an aminoglycoside phosphotransferase also conferring kanamycin resistance (Huang et al. 2002), and the cat gene encoding chloramphenicol acetyltransferase and conferring resistance to chloramphenicol ( $\mathrm{Li}$ et al. 2011). However, due to their substantially lower efficiency than aadAbased selection, they have not become widely adopted. It is also interesting to note that there are a number of markers that do not work for the primary selection of transplastomic cell lines, even though they confer good secondary resistance to the corresponding selection agents after their introduction into the plastid genome via selection for the aadA marker. These secondary markers include, for example, herbicide resistances (Ye et al. 2003) and resistances to toxic D-amino acids (Gisby et al. 2012). The reasons for these markers not being suitable for primary selection of transplastomic cells are not entirely clear, although lethality of the corresponding selection agents has been suggested as a possible explanation (Ye et al. 2003).

In summary, although the aadA gene provides a highly efficient and specific selectable marker, there is a need to develop alternative markers for plastid transformation to (a) extend the species range of the technology, and (b) facilitate the multistep engineering of plastid genomes, for example, by sequential introduction of multiple transgenes (supertransformation).

Aminoglycosides are a class of broad-spectrum antibiotics that inhibit prokaryotic translation through high-affinity binding to the small (30S) subunit of the 70S ribosome (Tenson and Mankin 2006). Bacteria can acquire resistance to aminoglycosides by enzymatic modification of the antibiotic molecules. Resistance-conferring, aminoglycosidemodifying enzymes are biochemically classified into (a) aminoglycoside $O$-nucleotidyltransferases (e.g., the AadA), (b) aminoglycoside $O$-phosphotransferases, and (3) aminoglycoside $\mathrm{N}$-acetyltransferases (Mingeot-Leclercq et al. 1999). During evolution and, presumably, under selective pressure from antibiotics present in the environment, a few bifunctional enzymes have arisen that harbor two aminoglycoside-modifying activities. These bifunctional enzymes are likely the result of gene fusion events and are thought to detoxify their host cells more efficiently than the monofunctional enzymes they evolved from. The bifunctional enzyme aminoglycoside acetyltransferase (6')-Ie/aminoglycoside phosphotransferase( (2")-Ia, AAC(6')-Ie/APH( (2")-Ia, is responsible for high-level antibiotic resistance in Grampositive bacteria, including pathogenic strains of Enterococcus and Staphylococcus (Frase et al. 2012). The enzyme comprises an N-terminal AAC $\left(6^{\prime}\right)$ domain (acetyltransferase domain) and a C-terminal $\mathrm{APH}\left(2^{\prime \prime}\right)$ domain (phosphotransferase domain; Fig. 1) that can function independently of each other. By phosphorylation and/or acetylation, the AAC $\left(6^{\prime}\right)-\mathrm{Ie} / \mathrm{APH}\left(2^{\prime \prime}\right)$-Ia enzyme (for brevity, subsequently referred to as AAC6-APH2) inactivates 


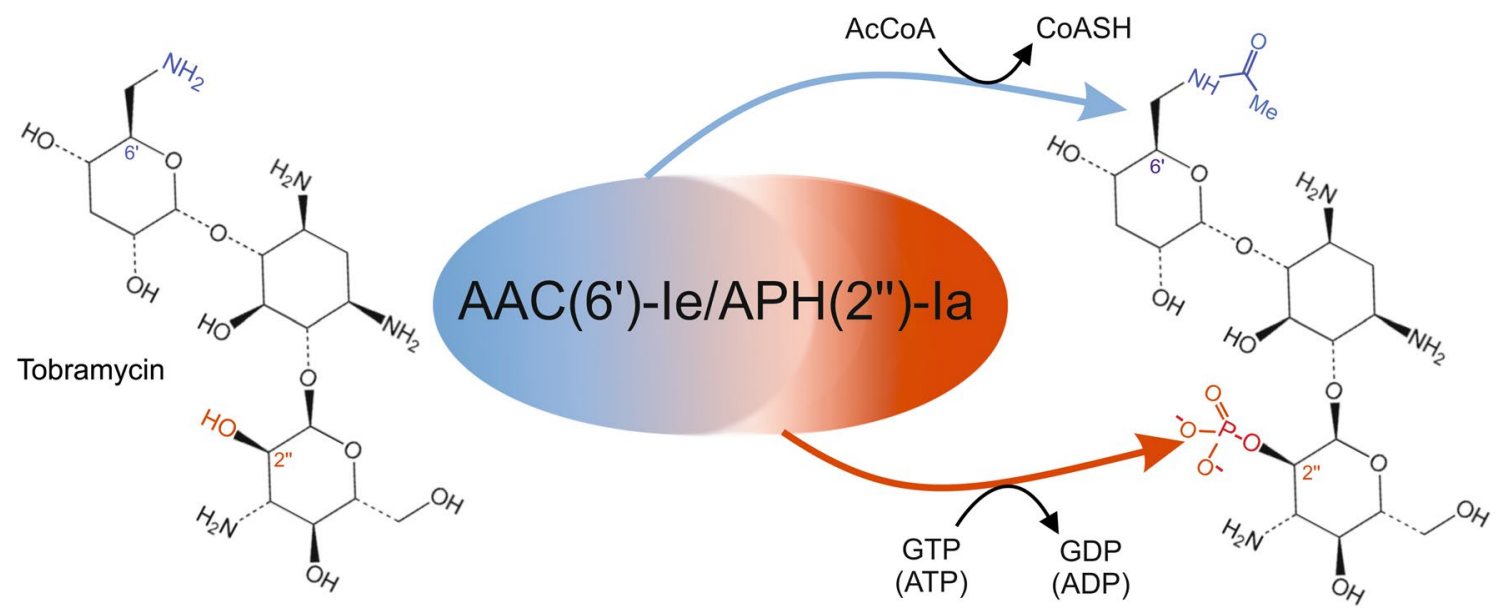

Fig. 1 The bifunctional $\mathrm{AAC}\left(6^{\prime}\right)-\mathrm{Ie} / \mathrm{APH}\left(2^{\prime \prime}\right)-\mathrm{Ia}$ enzyme has two active domains. The AAC $\left(6^{\prime}\right)$ domain catalyzes an acetylation reaction, using acetyl-CoA as the acetyl donor, to the 6'-amino group of ring I of aminoglycoside antibiotics. Exemplarily, the structure of

a broad range of aminoglycoside antibiotics. 4,6-disubstituted and so-called atypical aminoglycosides are particularly good substrates of its phosphorylation activity (Frase et al. 2012). 4,6-disubstituted aminoglycosides include, for example, kanamycin, tobramycin, amikacin, gentamicin $\mathrm{C}$ and sisomicin, whereas the 6-unsubstituted antibiotic neamine represents an atypical aminoglycoside.

Due to their bacterial origin, chloroplasts possess a prokaryotic translational apparatus that relies on $70 \mathrm{~S}$ ribosomes (Tiller and Bock 2014). Consequently, protein biosynthesis in chloroplasts displays similar antibiotic sensitivities as bacterial translation (Tenson and Mankin 2006; Bock 2015). Here, we have explored the possibility to use the gene for the bifunctional aminoglycoside-modifying enzyme AAC6-APH2 as a new selectable marker for plastid transformation in seed plants. We show that transplastomic lines can be obtained by selection for either tobramycin or gentamicin C (gentamicin). Importantly, in combination with tobramycin selection, the aac6-aph2 gene produces comparable numbers of transplastomic lines as the aadA marker, while giving no background of spontaneous antibiotic-resistant mutants.

\section{Materials and methods}

\section{Plant material and growth conditions}

Tobacco plants (Nicotiana tabacum cv. Petit Havana) were raised from seeds under aseptic conditions on agar-solidified (MS) medium (Murashige and Skoog 1962) containing $30 \mathrm{~g} / \mathrm{L}$ sucrose. For biolistic transformation, young leaves were harvested from 4-week-old plants. Regenerated tobramycin is shown here. The APH(2") domain uses GTP (or ATP) as phosphate donor and phosphorylates the 2 "-hydroxyl group of ring III of the aminoglycoside molecule (Frase et al. 2012)

transplastomic shoots were rooted and propagated on MS medium with $30 \mathrm{~g} / \mathrm{L}$ sucrose and $30 \mathrm{mg} / \mathrm{L}$ tobramycin sulfate (Duchefa or Sigma) or gentamicin sulfate (Duchefa). After rooting, homoplasmic plants were transferred to soil and grown under standard greenhouse conditions. To test for homoplasmy and maternal inheritance, seeds from appropriate crosses were germinated on MS medium containing $50 \mathrm{mg} / \mathrm{L}$ tobramycin sulfate or gentamicin sulfate.

\section{Vector construction}

pIT6 is a dual selectable chloroplast transformation vector that was constructed based on plastid transformation vector pKP9 (Zhou et al. 2008). The coding region of the bifunctional resistance gene $\operatorname{aac}\left(6^{\prime}\right)$-Ielaph( $\left.2^{\prime \prime}\right)-I a$ (subsequently abbreviated aac6-aph2; Frase et al. 2012) was codon optimized for the tobacco plastid genome and chemically synthesized (GeneArt, Regensburg, Germany) with the start codon being part of an NcoI restriction site and the stop codon followed by an $\mathrm{XbaI}$ site. The coding region was then subcloned as an $\mathrm{NcoI} / \mathrm{XbaI}$ fragment into a similarly cut chloroplast expression cassette consisting of the ribosomal RNA operon promoter (Nt Prrn), the T7 gene 10 leader sequence ( $G 10 \mathrm{~L}$, followed by the translation initiation codon as part of an NcoI restriction site; Zhou et al. 2008) and the $3^{\prime}$ UTR from the chloroplast rpsl6 gene (Nt Trps 16; Wurbs et al. 2007). The chimeric aac6-aph2 gene cassette was then cloned as a SacI/HindIII restriction fragment into vector $\mathrm{pKP} 9$, which contains the spectinomycin resistance gene aadA (Svab and Maliga 1993) as an additional selectable marker gene (Fig. 2; Table S1).

To be able to conduct transformation experiments with vectors containing only the aac6-aph2 gene cassette as 
Fig. 2 Generation of transplastomic tobacco plants by tobramycin selection. a Physical maps of the targeting region in the wild-type chloroplast genome (ptDNA) and the genomes of the transplastomic lines ( $N t$-IT) obtained with plastid transformation vectors pIT6, pIT19 and pIT20. Filled black boxes represent tobacco plastid genes, the aadA marker present in vector pIT6 is shown as an open box and the new aac6-aph 2 selectable marker (present in all pIT vectors) as a blue-red box. Expression elements (promoters, 5' UTRs and 3' UTRs) are represented as grey boxes and denoted by the source organism (Nt: Nicotiana tabacum; Cr: Chlamydomonas reinhardtii) and the source gene (Prrn: rRNA operon promoter; G10L: Shine-Dalgarno sequence from the bacteriophage T7 gene 10; Trps 16: 3' UTR from the plastid rps16 gene; SD rbcL: Shine-Dalgarno sequence from the plastid $r b c L$ gene; TpsbA: $3^{\prime}$ UTR from the plastid $p s b A$ gene; P $p s b A$ : promoter from the $p s b A$ gene; TrbcL: 3' UTR from the $r b c L$ gene). Genes above the lines are transcribed from left to right, genes below the lines are transcribed in the opposite direction. A PCR product derived from the tobacco $p s a B$ gene was used as probe in restriction fragment length polymorphism (RFLP) analyses and is represented as a black bar. The expected sizes of plastid DNA fragments in RFLP analyses with the restriction enzyme BgIII are indicated below each map. b RFLP analysis of transplastomic lines. DNA samples of the wild type (wt) and several independently generated antibiotic-resistant lines obtained from primary selection or the first regeneration round were digested with BglII, separated in $1 \%$ agarose gels, blotted and hybridized to the radiolabeled psaB probe shown in panel (a). The probe detects the expected $3.5 \mathrm{~kb}$ fragment in the wild type, a $6.8 \mathrm{~kb}$ fragment in the transplastomic lines obtained with vector pIT6, and a 5.8 and $5.7 \mathrm{~kb}$ fragment in the transplastomic lines produced with vectors pIT19 and pIT20, respectively

selectable marker and to avoid flip-flop recombination between the two Nt Prrn promoter copies present in pIT6 (Rogalski et al. 2006), chloroplast transformation vectors pIT19 and pIT20 were constructed. In pIT19, the aac6$a p h 2$ gene is driven by the $p s b A$ gene promoter $(\mathrm{P} p s b A)$ from Chlamydomonas reinhardtii ( $C r \mathrm{P} p s b A$; Fleischmann et al. 2011; Table S1). In pIT20, the aac6-aph2 gene is under the control of the tobacco ribosomal RNA operon promoter (Nt Prrn). In both vectors, the 3' UTR from the $C$. reinhardtii plastid $r b c L$ gene $(C r$ TrbcL; Zou et al. 2003) was used as terminator for the marker gene cassette (Fig. 2). pIT19 was constructed by PCR amplification of the aac6-aph2 coding region, followed by cloning as a NcoI/SphI restriction fragment into the identically cut plasmid pDK305. pDK305 is a derivative of plastid transformation vector pRB95 (Ruf et al. 2001) and contains a chimeric aadA gene driven by the $C r$ P $p s b A$ promoter and the $C r \mathrm{~T} r b c L$ terminator. For construction of pIT20, the aac6-aph2 gene together with the ribosomal RNA operon promoter (Nt Prrn) and the T7 gene 10 leader sequence was amplified and cloned as XhoI/SphI restriction fragment into the $\mathrm{XhoI} / \mathrm{SphI}$-digested plasmid pIT19. The DNA sequences of all coding regions and expression elements (promoters, 5' UTRs and 3' UTRs) used in this study are given in Table S1.
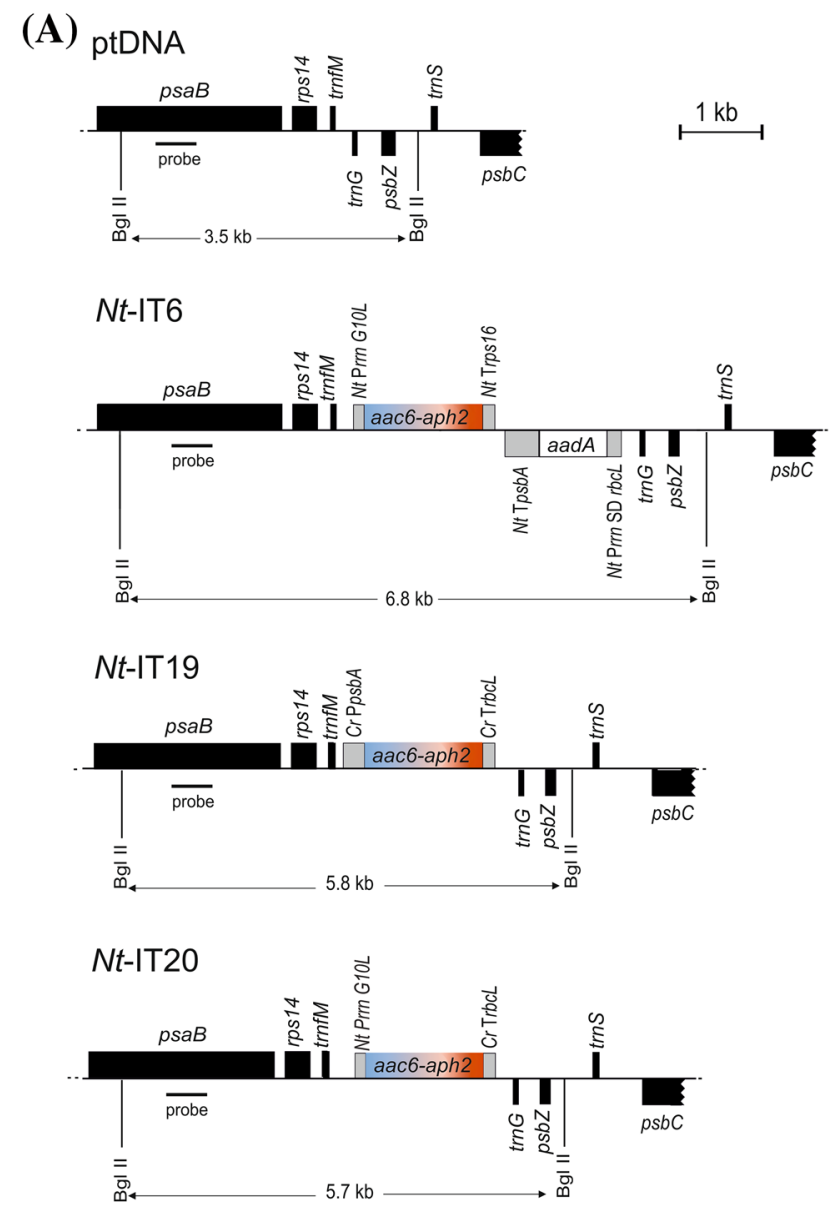

(B)

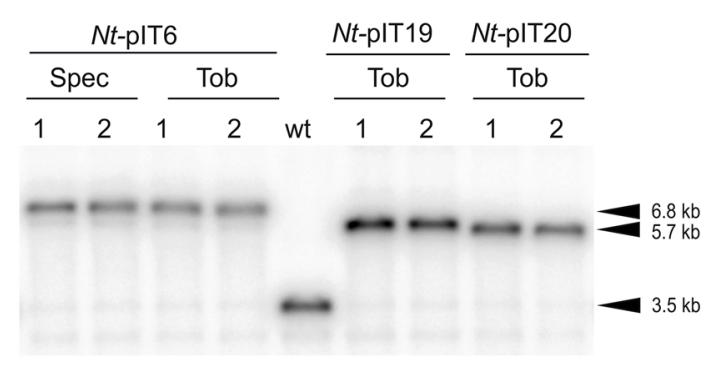

\section{Plastid transformation and selection of homoplasmic transplastomic lines}

For chloroplast transformation, young leaves harvested from aseptically grown tobacco plants were biolistically bombarded with plasmid DNA (pIT6, pIT19, pIT20 and pDK305) coated onto $0.6 \mu \mathrm{m}$ gold particles (BioRad) using the DuPont PDS-1000/He biolistic gun with the hepta adaptor setup. After bombardment, the leaves were cut into small pieces $(\sim 5 \times 5 \mathrm{~mm}$ in size $)$ which were then placed onto the surface of an MS-based selective regeneration medium containing tobramycin sulfate $(30,40$ or $50 \mathrm{mg} / \mathrm{L})$ or gentamicin sulfate $(50 \mathrm{mg} / \mathrm{L})$. Spectinomycinresistant shoots were selected on medium with $500 \mathrm{mg} / \mathrm{L}$ 
spectinomycin (Svab and Maliga 1993). Selection was conducted under $25 \mu \mathrm{E} \mathrm{m}^{-2} \mathrm{~s}^{-1}$ light intensity in a $16 \mathrm{~h}$ light/ $8 \mathrm{~h}$ dark cycle. When a medium change was performed, the leaf pieces were transferred to fresh selection medium after 3-4 weeks. Transplastomic lines selected on spectinomycin medium were confirmed by an additional regeneration round on medium containing $500 \mathrm{mg} / \mathrm{L}$ streptomycin (Bock 2001). Primary resistant lines in the spectinomycin selection system typically appeared after 4-10 weeks, primary tobramycin or gentamicin-resistant lines appeared after 10-18 weeks. Antibiotic-resistant calli or leaf pieces from regenerating shoots were transferred to fresh selection medium for further propagation and purification of homoplasmic transplastomic tissue.

For quantification of transformation efficiencies, primary resistant lines and confirmed transplastomic lines were counted (Table 1). Transformation frequencies were expressed as number of transformants divided by the number of selected explants. It should be noted that, in plastid transformation, there can be substantial variation in transformation efficiency between experiments. The source(s) of this variation are currently not fully understood, but it is believed that a number of factors contribute, including the physiological status of the bombarded leaf material, the quality of the gold particle preparation and subtle variations in the many parameters involved in tissue culture and selection.

\section{Crosses and inheritance assays}

To confirm the homoplasmic state of transplastomic lines and maternal transgene inheritance, plants were grown to maturity under standard greenhouse conditions. Upon flowering, plants were either self-pollinated or reciprocally crossed to wild-type plants. Seeds were harvested and assayed by germination on tobramycin-containing (50 $\mathrm{mg} / \mathrm{L}$ tobramycin sulfate) or gentamicin containing MS medium ( $50 \mathrm{mg} / \mathrm{L}$ gentamicin sulfate).

\section{Isolation of nucleic acids and DNA gel blot analyses}

Total genomic DNA was isolated from fresh leaf material using a cetyltrimethylammoniumbromide (CTAB)-based protocol (Doyle and Doyle 1990).

For Southern blot analyses, DNA samples were digested with the restriction enzyme BglII, separated by gel electrophoresis in $1 \%(\mathrm{w} / \mathrm{v})$ agarose gels and transferred onto Hybond XL nylon membranes (GE Healthcare) by capillary blotting using a standard protocol. As RFLP probe, a 550 bp PCR amplicon derived from the psaB coding region (Fig. 2a) was used (Wurbs et al. 2007). The amplified fragment was purified by agarose gel electrophoresis and extraction from the excised gel slice using the GFX
PCR (DNA and Gel Band Purification) kit. Radiolabelling was performed with $\left[\alpha^{32} \mathrm{P}\right] \mathrm{dCTP}$ by random priming (Multiprime DNA labeling system, GE Healthcare) according to the protocol of the supplier. Hybridizations were performed overnight at $65^{\circ} \mathrm{C}$. Following standard washing steps, the membranes were exposed to autoradiographic screens and then scanned in a Typhoon TRIO+scanner (GE Healthcare).

\section{Results}

Construction of plastid transformation vectors based on the bifunctional aac6-aph2 resistance marker and identification of suitable selection conditions for chloroplast transformation.

To explore the possibility to use the bifunctional aac6$a p h 2$ gene as a selectable marker for chloroplast transformation, we first tested the sensitivities of tobacco leaf explants to those aminoglycosides that are efficiently detoxified by the AAC6-APH2 enzyme in bacteria (Frase et al. 2012). The following antibiotics were included in these assays: G418 (also known as geneticin), gentamicin, tobramycin, sisomicin, kanamycin B and kanamycin A. Tobacco cells turned out to be sensitive to all of these drugs, albeit the minimum antibiotic concentration required to fully suppress plant regeneration differed between the tested aminoglycosides (Fig. S1). For example, while G418 applied at a concentration of $5 \mathrm{mg} / \mathrm{L}$ was sufficient to cause rapid bleaching of the leaf explants and inhibit shoot regeneration, tobramycin needed to be applied at an approximately tenfold higher concentration to achieve complete suppression of callus growth and regeneration (Fig. S1). However, previous work on selectable marker development for plastids has revealed that high antibiotic sensitivity is not necessarily correlated to the efficiency of selection for transplastomic clones. For example, in the currently most efficient transplastomic selection system (based on spectionomycin resistance conferred by chimeric aadA genes), typically antibiotic concentrations of as much as $500 \mathrm{mg} / \mathrm{L}$ are used to prevent the occasional regeneration of nonresistant plants (escapees). In fact, nonlethal selection has been proposed to be advantageous in plastid transformation in that it allows the transformed cells exposed to the selection agent to survive for a sufficiently long time to establish the antibiotic resistance (Ye et al. 2003). Based on these considerations, we chose tobramycin, sisomicin and gentamicin as selection agents to be tested in chloroplast transformation experiments.

To be able to conduct chloroplast transformation experiments with the bifunctional aac6-aph2 gene as selectable marker, a set of plastid transformation vectors was constructed (see "Materials and methods"; Fig. 2). Vector pIT6 


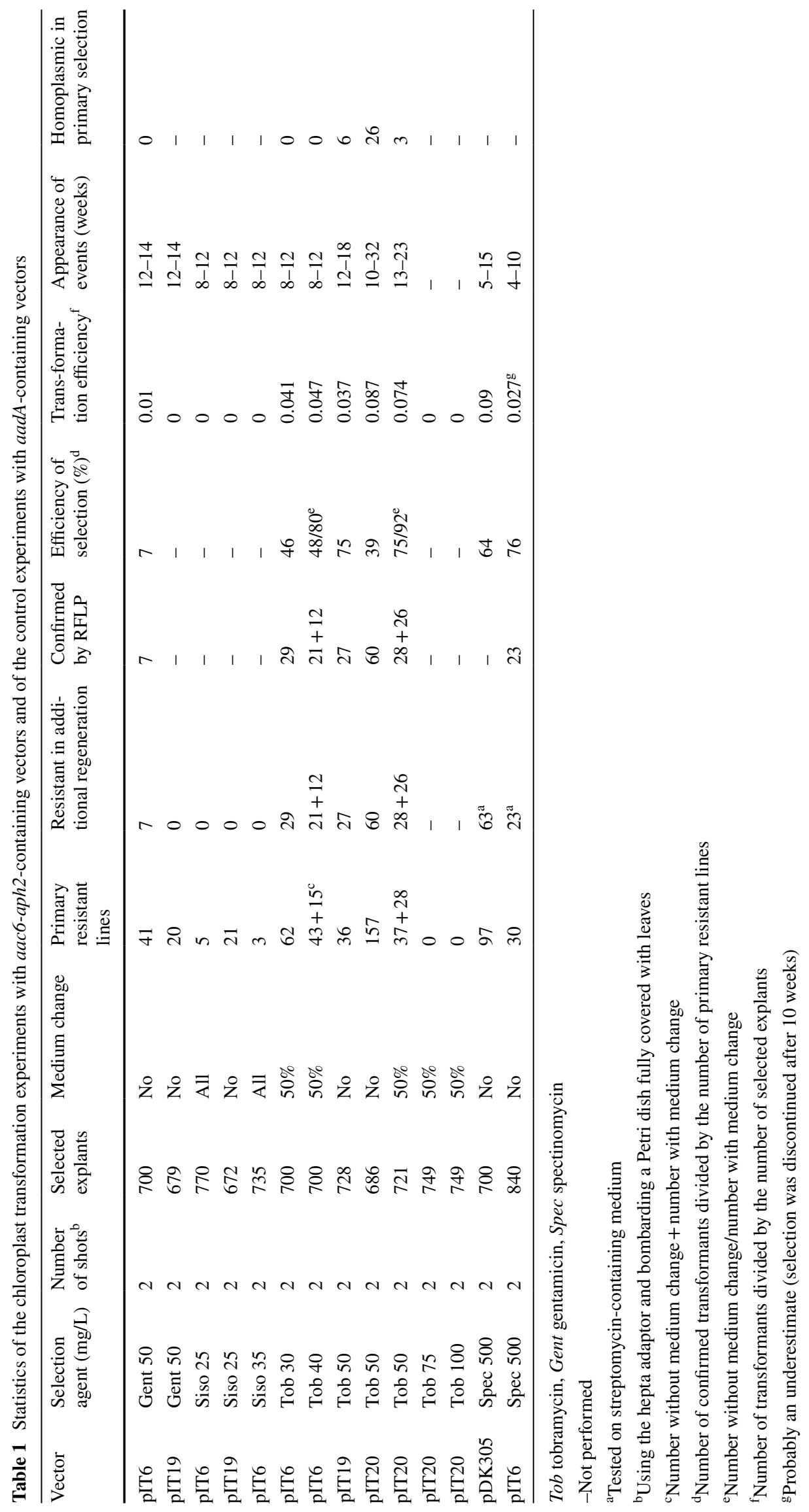


was built to test whether the aac6-aph2 gene is capable of conferring resistance upon expression from the plastid genome. It contains the aadA marker in addition to the aac6-aph2 gene and, therefore, allows for introduction of the aac6-aph2 gene into the chloroplast genome by standard spectinomycin selection. In addition, the use of this vector facilitates side-by-side comparison between the standard aadA-based spectinomycin selection of transplastomic lines (Svab and Maliga 1993) and any new selection scheme potentially based on aac6-aph2. By contrast, vectors pIT19 and pIT20 were designed for primary selection for AAC6-APH2-based antibiotic resistances (Fig. 2a). They contain the aac6-aph2 gene as the sole selectable marker gene and only differ in the expression signals driving aac6-aph2 expression. While, in vector pIT20, the aac6-aph2 is under the control of the strongest known expression signals for tobacco chloroplasts (a fusion of the ribosomal RNA operon promoter to a synthetic ShineDalgarno sequence derived from the bacteriophage T7 gene 10 leader sequence; Kuroda and Maliga 2001; Oey et al. 2009a), in vector pIT19, the marker gene is driven by a considerably weaker promoter and $5^{\prime}$ UTR (derived from the $p s b A$ gene of the unicellular green alga Chlamydomonas reinhardtii; Emadpour et al. 2015).

\section{Plastid transformation using aac6-aph2 as selectable marker gene}

Chloroplast transformation experiments were conducted with all three vectors using the biolistic protocol (Svab et al. 1990; Svab and Maliga 1993) and tobramycin, sisomicin and gentamicin as selection agents (Table 1). To allow for comparison of selection efficiencies, part of the samples bombarded with the aadA-containing vector pIT6 were subjected to selection for spectinomycin resistance. As a control, a standard aadA-based plastid transformation vector (pDK305; see "Materials and methods"; Ruf et al. 2001) was also included in the experiments.

Introduction of the aac6-aph2 gene as a passenger gene in transformation experiments with vector pIT6 followed by selection for spectinomycin resistance (Figs. 2b, 3 ) allowed us to determine the resistance levels of transplastomic lines to tobramycin, sisomicin and gentamicin. Based on regeneration tests on different concentrations of these three antibiotics and the previously performed antibiotic sensitivity tests of wild-type plants (Fig. S1), 30, 40 and $50 \mathrm{mg} / \mathrm{L}$ tobramycin, 25 and $35 \mathrm{mg} / \mathrm{L}$ sisomicin, and $50 \mathrm{mg} / \mathrm{L}$ gentamicin were chosen as concentrations for selection of transplastomic lines in initial plastid transformation experiments.

Selection for sisomicin resistance gave a number of regenerants ('Primary resistant lines'; Table 1). However, additional regeneration rounds clearly showed that these putative lines were as sensitive to sisomicin as the wild type, whereas transplastomic Nt-IT6 controls lines that had been isolated by spectinomycin selection were resistant to 25 and $35 \mathrm{mg} / \mathrm{L}$ sisomicin. These results strongly suggested that all primary regenerants obtained by sisomicin selection are escapees rather than true transplastomic lines (Table 1).

In two independent transformation experiments, the bifunctional aac6-aph2 resistance gene was combined with selection for gentamicin resistance. While one of the experiments produced only false positive lines, seven transplastomic lines were isolated from the second experiment and confirmed by both RFLP analysis and additional regeneration rounds on gentamicin-containing media (Fig. 3). However, the overall transformation efficiency obtained with gentamicin as selection agent was rather low and did not come close to the efficiency reached with the aadA and spectinomycin selection (Table 1).

By contrast, selection for tobramycin resistance yielded a large number of resistant lines, many of which were confirmed as true transplastomic events by their sustained resistance in subsequent regeneration rounds and by RFLP analyses (Figs. 2b, 3; Table 1). Transplastomic lines were recovered from all three antibiotic concentrations tested (30, 40 and $50 \mathrm{mg} / \mathrm{L}$ tobramycin). As expected, the number of false positive events (escapees) was higher on the low antibiotic concentrations (Table 1). Also, the vector with the strong expression signals driving the aac6-aph2 selectable marker (pIT20) produced more transplastomic lines than the vector with the weaker expression signals (pIT19; Table 1).

To test if the background of false positive events can be eliminated by selecting for higher concentrations of tobramycin, transformation experiments with selection for 75 or $100 \mathrm{mg} / \mathrm{L}$ tobramycin were conducted. These experiments did not produce any resistant lines indicating that $30-50 \mathrm{mg} / \mathrm{L}$ is the suitable selection window for tobramycin (Table 1). To test whether the background regeneration can be attributed to the instability of tobramycin, the effect of a medium change was analyzed. To this end, the leaf pieces were transferred to fresh selection medium after 3-4 weeks of incubation. Indeed, this protocol substantially reduced background regeneration and increased selection efficiency (Table 1). In addition, the reexposure of primary regenerants to selection medium in a second regeneration round (Fig. 3) turned out to be a very efficient method of distinguishing escapees from true transformants, in that all candidate lines surviving this selection were transplastomic (Table 1).

Overall, the transformation efficiency with aac6-aph2 as selectable marker in combination with tobramycin selection was similarly high as that with the standard marker gene aadA and spectinomycin selection (Table 1), thus providing 


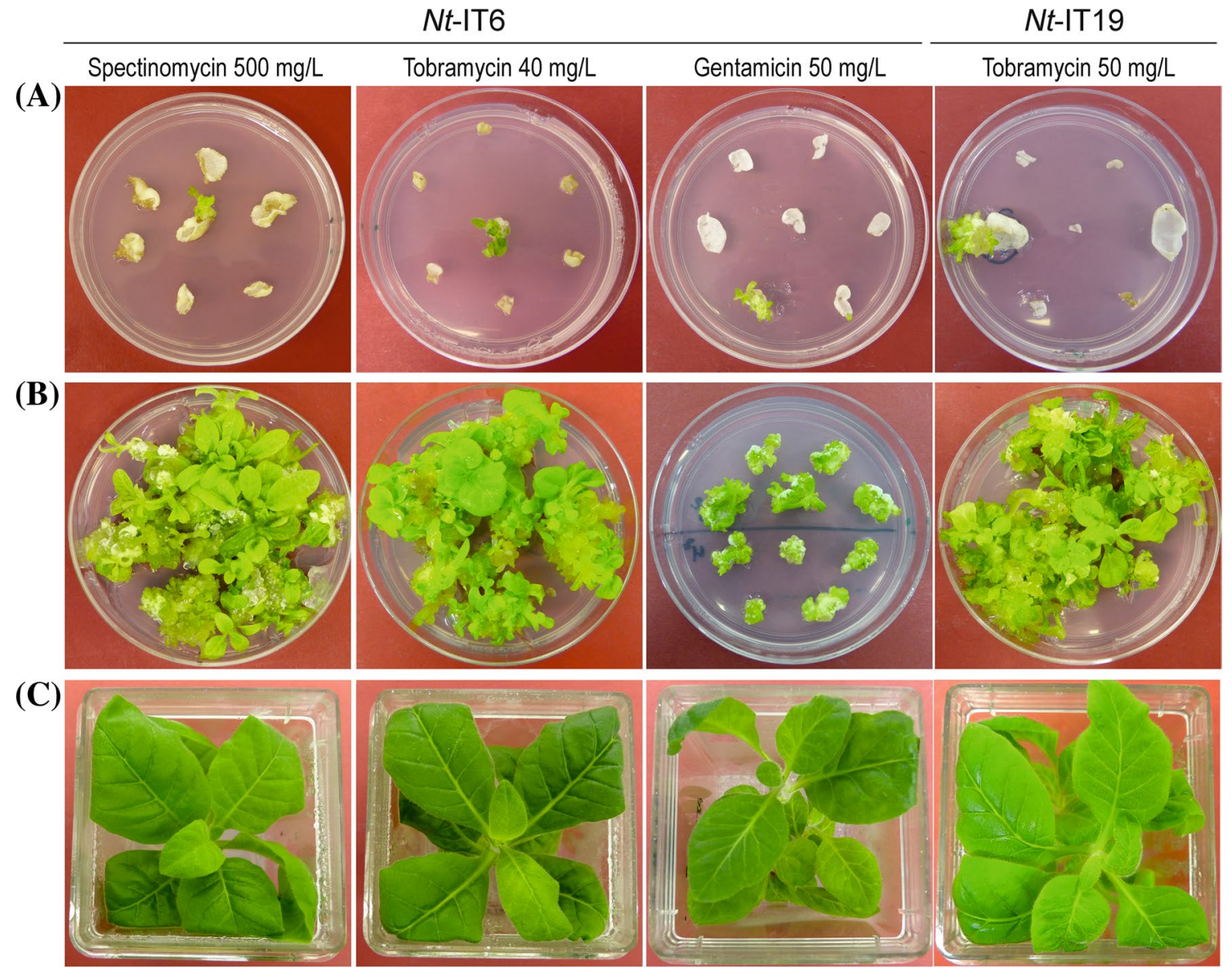

Fig. 3 Generation of transplastomic tobacco lines by selection for tobramycin resistance or gentamicin resistance. a Primary selection of transplastomic lines on medium with tobramycin or gentamicin. The lines were produced with vectors pIT6 and pIT19, respectively (see Fig. 2). As a control, a transplastomic Nt-IT6 clone obtained by selection for spectinomycin resistance is also shown. b Additional regeneration rounds conducted in the presence of the selection agent to obtain homoplasmic transplastomic shoots. Nt-IT6 plates were photographed after 4 weeks, the $N t$-IT19 plate after 6 weeks. c Rooting and growth of transplastomic lines under aseptic conditions. Tobramycin or gentamicin-resistant shoots from selection plates were grown on phytohormone-free medium in the presence of the antibiotic (30 $\mathrm{mg} / \mathrm{L}$ tobramycin or gentamicin), the spectinomycin-resistant plant was grown in the presence of $500 \mathrm{mg} / \mathrm{L}$ spectinomycin an attractive alternative selection system for transplastomic plants.

\section{Characterization of transplastomic plants generated with aac6-aph2 as selectable marker gene}

Transplastomic line containing the aac6-aph2 marker gene grew normally both under sterile conditions (Fig. 3c) and in the greenhouse (Fig. 4a). Plants grown in the greenhouse were phenotypically indistinguishable from wild-type plants (Fig. 4), were fertile and produced normal amounts of seeds.

To ultimately confirm homoplasmy of the transplastomic lines and to demonstrate maternal inheritance of the aac6-aph 2 marker, seed assays were conducted. To this end, reciprocal crosses between transplastomic plants and wild-type plants were conducted and the resulting seeds were germinated in the presence of tobramycin (Fig. 4b). As expected, the tobramycin resistance was stably transmitted into the next generation and displayed strictly maternal inheritance, as typical of plastid-encoded traits. Absence of tobramycin-sensitive seedlings from the progeny also ultimately verified homoplasmy of the transplastomic plants (Fig. 4b). Identical results were obtained when gentamicin resistance was tested by inheritance assays (Fig. S2).

Isolation of homoplasmic transplastomic lines with the established spectinomycin selection system based on the aadA marker gene typically requires two or three additional rounds of regeneration under antibiotic selection to eliminate residual copies of the wild-type plastid genome (Svab and Maliga 1993). When the primary transplastomic regenerants from selection for $50 \mathrm{mg} / \mathrm{L}$ tobramycin 
(A)

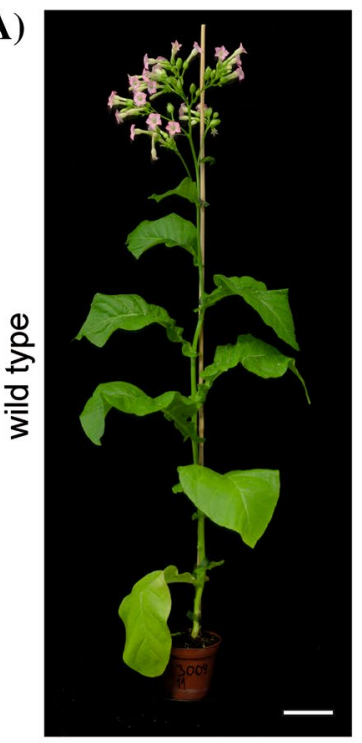

(B)

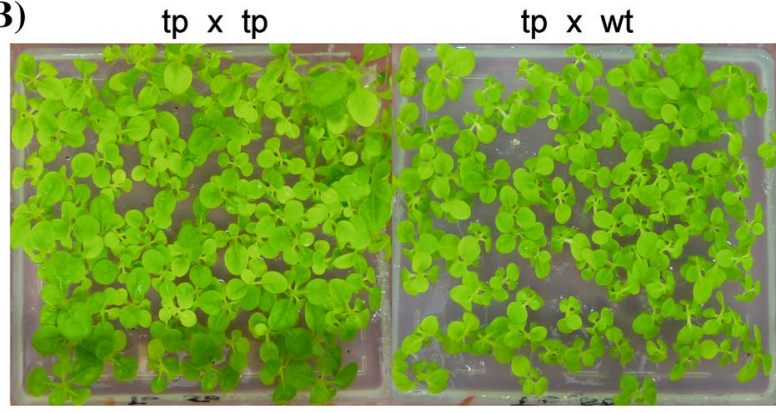

wt $x$ tp

wt $\mathrm{x}$ wt

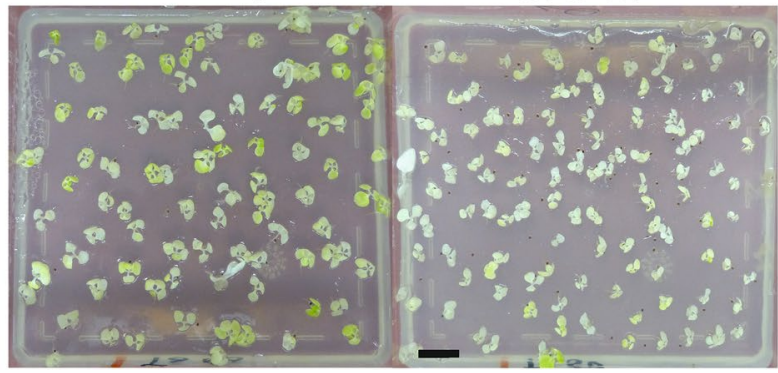

Fig. 4 Growth of transplastomic plants to maturity and seed assays confirming maternal transgene inheritance. a Transplastomic plants with the inserted aac6-aph2 marker gene (right) grow like wild-type plants (left) under greenhouse conditions. Scale bars $10 \mathrm{~cm}$. b Seed assays to confirm stable maternal inheritance of the chloroplastencoded tobramycin resistance gene. A transplastomic Nt-pIT6 plant (tp) was selfed (tp $\times$ tp) and reciprocally crossed to a wild-type plant $(\mathrm{tp} \times w t, w t \times t p)$. As a control for antibiotic sensitivity, the selfed wild type (wt $\times w t)$ was also included. While the progeny from all crosses with the transplastomic line as maternal parent are homogeneously resistant to tobramycin $(50 \mathrm{mg} / \mathrm{L})$, the progeny from crosses with a wild-type plant as maternal parent are uniformly sensitive to tobramycin. Scale bar $1 \mathrm{~cm}$

were analyzed by Southern blotting, we noticed that a high proportion of them was already homoplasmic (Fig. 2c; Table 1). This was not the case with the transplastomic lines isolated from selection for lower levels of tobramycin resistance (30 or $40 \mathrm{mg} / \mathrm{L}$; Table 1) indicating that the strength of the selection pressure is responsible for this effect. Thus, although the primary selection of transplastomic lines with tobramycin takes, on average, longer than selection with spectinomycin (Table 1), considerable time is saved by faster attainment of homoplasmy.

\section{Cross-resistances to aminoglycoside antibiotics}

The successful generation of transplastomic lines with the three different aac6-aph2 vectors (Fig. 2) allowed us to determine the resistance levels to different aminoglycoside antibiotics and compare them to the wild type and to transplastomic plants generated with the standard selectable marker gene aadA. To this end, leaf explants of homoplasmic transplastomic plants were exposed to tobramycin, gentamicin, tobramycin + gentamicin, spectinomycin and kanamycin A. As expected, strong spectinomycin resistance was observed in $N t$-DK305 and Nt-IT6 plants that harbor the aadA marker in their plastid genomes (Fig. 5). By contrast, $N t$-IT19 and $N t$-IT20 plants displayed no appreciable resistance to spectinomycin, demonstrating that the AAC6-APH2 enzyme cannot detoxify spectinomycin.

All transplastomic plants containing the aac6-aph2 marker ( $N t$-IT6, $N t$-IT19 and $N t$-IT20) were capable of regenerating in the presence of tobramycin. However, regeneration was noticeably faster in plants that express the aac6-aph2 gene from the strong ribosomal RNA operon promoter and the strongest known Shine-Dalgarno sequence ( $G 10 \mathrm{~L} ; \mathrm{Nt}$-IT6 and $N t$-IT20) than in plants where expression is driven by the considerably weaker heterologous $p s b A$ promoter and $5^{\prime}$ untranslated region from Chlamydomonas (Nt-IT19; Fig. 5; Fig. S3). This difference was even more pronounced when regeneration assays were performed in the presence of both tobramycin and gentamicin $(50 \mathrm{mg} / \mathrm{L}$ each). This strong selection pressure substantially delayed regeneration in all transplastomic lines harboring the aac6-aph2 transgene, but much more so in $N t$ IT19 plants than in the $N t$-IT6 and $N t$-IT20 plants (Fig. 5; Fig. S3).

Finally, we also assayed all transplastomic lines for cross-resistance to kanamycin A. While the aac6-aph2 gene did not confer increased tolerance to spectinomycin, it provided some resistance to kanamycin (Fig. 5). However, although kanamycin belongs to the 4,6-disubstituted aminoglycosides (like tobramycin and gentamicin) and was reported to be efficiently detoxified by the AAC6-APH2 enzyme in bacteria (Frase et al. 2012), the resistance level in all transplastomic $N t$-IT lines was lower compared to tobramycin and gentamicin (Fig. 5), indicating that the aac6-aph2 marker is not ideal for kanamycin selection of transplastomic plants. 

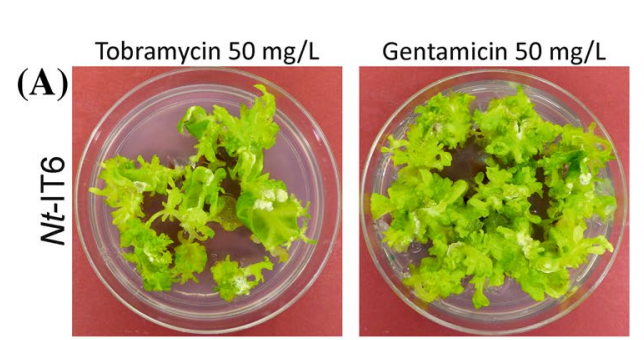

Tobramycin $50 \mathrm{mg} / \mathrm{L}+$
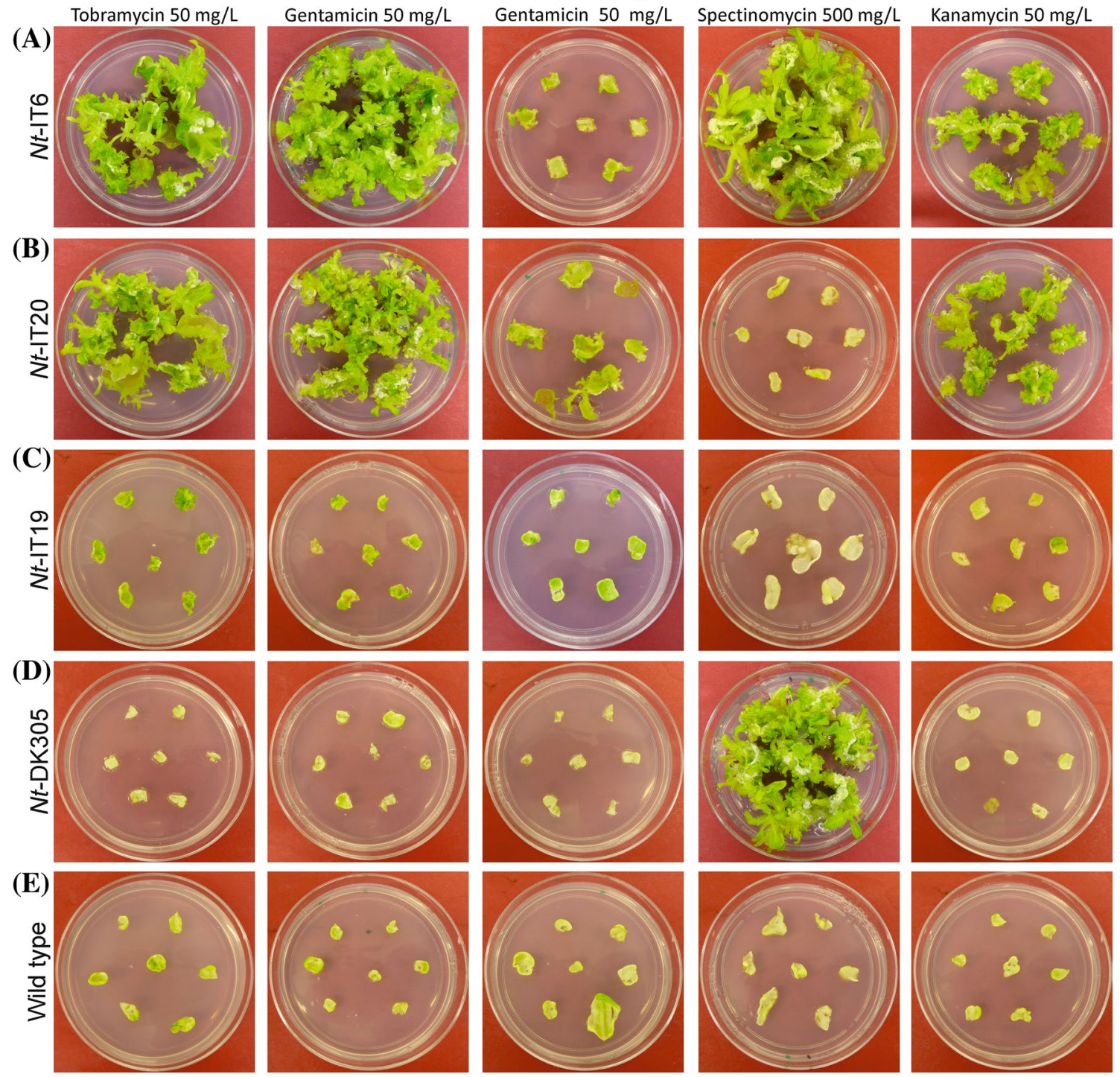

Fig. 5 Specificity test of the aadA and aac6-aph 2 marker genes. The detoxification activity of the encoded enzymes towards the four aminoglycoside antibiotics tobramycin, gentamicin, spectinomycin and kanamycin was assayed by exposing leaf explants of transplastomic plants and a wild-type control to selective regeneration medium containing tobramycin, gentamicin, tobramycin + gentamicin, spectinomycin or kanamycin. Photographs were taken after 3 weeks. a An $N t$-IT6 line harboring the aac6-aph2 gene under the control of strong

\section{Discussion}

In the course of this work, we have developed a novel selection marker for plastid transformation. The marker gene encodes a bifunctional enzyme that has both phosphotransferase and acetyltransferase activity (Fig. 1) towards a broad range of aminoglycoside antibiotics. We have shown that, when tethered to plastid expression signals, the aac6-aph2 gene provides sufficiently strong resistance to tobramycin and gentamicin to select plastid transformants expression elements and additionally the aadA marker. b An NtIT20 line containing the aac6-aph2 gene under the control of strong expression elements. c An Nt-IT19 line harboring the aac6-aph2 gene under the control of weak expression elements. d An Nt-DK305 line expressing only the aadA marker gene. e A wild-type plant. Note that transplastomic lines containing only the aadA gene (Nt-DK305) cannot detoxify tobramycin and gentamicin. See also Fig. S3

in tobacco. The homoplasmic transplastomic status of the selected lines was evidenced by RFLP analyses and inheritance assays (Figs. 2, 4; Table 1). Tobramycin selection appears to be superior to gentamicin selection (Table 1), and we, therefore, recommend to use the aac6-aph2 marker in combination with selection for either 40 or $50 \mathrm{mg} / \mathrm{L}$ tobramycin.

The aac6-aph 2 marker provides an alternative selectable marker gene to the commonly used spectinomycin resistance gene aadA. The efficiency of both marker genes 
(number of transplastomic lines obtained per bombarded leaf sample) is very similar (Table 1). When comparing the standard aadA-based spectinomycin selection system with the aac6-aph2-based tobramycin selection, each system has potential advantages and disadvantages. The advantages of the aac6-aph2 marker lie in the absence of spontaneous antibiotic-resistance mutants that frequently appear in spectinomycin selection for transplastomic lines (Svab et al. 1990; Svab and Maliga 1993, 1991; Bock 2001), and the faster attainment of homoplasmy in that a substantial number of primary transplastomic lines are already homoplasmic (Table 1). The latter may be attributable to the stronger selection pressure exerted by tobramycin selection compared to spectinomycin selection. This explanation is consistent with the narrower selection window for tobramycin, with tobramycin concentrations of $75 \mathrm{mg} / \mathrm{L}$ and above preventing selection of transplastomic cells (Table 1). Current disadvantages of the aac6-aph2 marker lies in the somewhat longer primary selection phase (Table 1) which, however, is (over)compensated by the quicker attainment of homoplasmy, and the appearance of some escapees (i.e., regenerating plantlets that are not tobramycin resistant). Their frequency can be substantially reduced by a medium change during primary selection of transplastomic clones (Table 1). However, given the labor and cost involved in a complete medium change, it may be more practical to simply eliminate the escapees by an additional regeneration test on tobramycin-containing medium. The latter procedure reliably distinguished true transformants from escapees in that all lines that were resistant to tobramycin in an additional regeneration assay turned out to be true plastid transformants (Table 1).

New selection markers for chloroplast transformation are an important addition to our toolbox for plastid genome engineering for two reasons. First, they facilitate supertransformation of the plastid genome, that is the transformation of an already transplastomic plant with a second constructs, without the need for prior selectable marker recycling (Lutz and Maliga 2007; Day and Goldschmidt-Clermont 2011). Supertransformation is not only the method of choice for the successive introduction of multiple transgenes in multigene engineering efforts, it also facilitates the construction of multiple knock-outs or the site-directed mutagenesis of two unlinked genes in the plastid genome (Ehrnthaler et al. 2014). Secondly, new selectable marker genes will likely be instrumental in the extension of the plastid transformation technology to new species. The lack of efficient selection systems represents the major obstacle to the implementation of plastid transformation in monocot species, including cereals as the world's most important food crops. Since most, if not all, cereal species are endogenously resistant to spectinomycin (Fromm et al. 1987), a breakthrough with plastid transformation in monocots will be critically dependent on the identification of selection agents that effectively inhibit callus growth in the dark (Ahmadabadi et al. 2007). Experiments are underway to assess the sensitivity of cereal cell cultures to tobramycin and gentamicin and test the aac6-aph2 gene in combination with optimized expression signals for non-green plastids (Zhang et al. 2012; Caroca et al. 2013) for its suitability as a selectable marker for monocot plastid transformation. Preliminary experiments indicate that maize callus growth is sensitive to both tobramycin and gentamicin.

Acknowledgements Open access funding provided by Max Planck Society. We thank Pierre Endries and Marta Maria Bednarska (both MPI-MP) for excellent technical assistance. I.T. was the recipient of a $\mathrm{PhD}$ fellowship from the Ministry of Science, Research and Technology of Iran. This work was supported by a grant from the European Research Council (ERC) under the European Union's Horizon 2020 research and innovation programme (ERC-ADG-2014; Grant Agreement No 669982) to R.B. and by the Max Planck Society.

Author contributions IT designed and performed research and analyzed data. SR designed research and analyzed data. RB conceived of the study, designed research, analyzed data and wrote the paper with input from all co-authors.

Open Access This article is distributed under the terms of the Creative Commons Attribution 4.0 International License (http:// creativecommons.org/licenses/by/4.0/), which permits unrestricted use, distribution, and reproduction in any medium, provided you give appropriate credit to the original author(s) and the source, provide a link to the Creative Commons license, and indicate if changes were made.

\section{References}

Ahmadabadi M, Ruf S, Bock R (2007) A leaf-based regeneration and transformation system for maize (Zea mays L.) Transgenic Res 16:437-448

Apel W, Bock R (2009) Enhancement of carotenoid biosynthesis in transplastomic tomatoes by induced lycopene-to-provitamin A conversion. Plant Physiol 151:59-66

Bock R (2001) Transgenic plastids in basic research and plant biotechnology. J Mol Biol 312:425-438

Bock R (2014) Genetic engineering of the chloroplast: novel tools and new applications. Curr Opin Biotechnol 26:7-13

Bock R (2015) Engineering plastid genomes: methods, tools, and applications in basic research and biotechnology. Annu Rev Plant Biol 66:211-241

Bock R, Koop H-U (1997) Extraplastidic site-specific factors mediate RNA editing in chloroplasts. EMBO J 16:3282-3288

Bohmert-Tatarev K, McAvoy S, Daughtry S, Peoples OP, Snell KD (2011) High levels of bioplastic are produced in fertile transplastomic tobacco plants engineered with a synthetic operon for the production of polyhydroxybutyrate. Plant Physiol 155:1690-1708

Boynton JE, Gillham NW, Harris EH, Hosler JP, Johnson AM, Jones AR, Randolph-Anderson BL, Robertson D, Klein TM, Shark KB, Sanford JC (1988) Chloroplast transformation in Chlamydomonas with high velocity microprojectiles. Science 240:1534-1538 
Caroca R, Howell KA, Hasse C, Ruf S, Bock R (2013) Design of chimeric expression elements that confer high-level gene activity in chromoplasts. Plant J 73:368-379

Carrer H, Hockenberry TN, Svab Z, Maliga P (1993) Kanamycin resistance as a selectable marker for plastid transformation in tobacco. Mol Gen Genet 241:49-56

Day A, Goldschmidt-Clermont M (2011) The chloroplast transformation toolbox: selectable markers and marker removal. Plant Biotechnol J 9:540-553

De Cosa B, Moar W, Lee S-B, Miller M, Daniell H (2001) Overexpression of the $\mathrm{Bt}$ cry2Aa2 operon in chloroplasts leads to formation of insecticidal crystals. Nature Biotechnol 19:71-74

Doyle JJ, Doyle JL (1990) Isolation of plant DNA from fresh tissue. Focus 12:13-15

Ehrnthaler M, Scharff LB, Fleischmann TT, Hasse C, Ruf S, Bock R (2014) Synthetic lethality in the tobacco plastid ribosome and its rescue at elevated growth temperatures. Plant Cell 26:765-776

Emadpour M, Karcher D, Bock R (2015) Boosting riboswitch efficiency by RNA amplification. Nucleic Acids Res 43:e66

Fleischmann TT, Scharff LB, Alkatib S, Hasdorf S, Schöttler MA, Bock R (2011) Nonessential plastid-encoded ribosomal proteins in tobacco: a developmental role for plastid translation and implications for reductive genome evolution. Plant Cell 23:3137-3155

Frase H, Toth M, Vakulenko SB (2012) Revisiting the nucleotide and aminoglycoside substrate specificity of the bifunctional aminoglycoside acetyltransferase $\left(6^{\prime}\right)$-le/aminoglycoside phosphotransferase(2")-la enzyme. J Biol Chem 287:43262-43269

Fromm H, Edelman M, Aviv D, Galun E (1987) The molecular basis for rRNA-dependent spectinomycin resistance in Nicotiana chloroplasts. EMBO J 6:3233-3237

Fuentes P, Zhou F, Erban A, Karcher D, Kopka J, Bock R (2016) A new synthetic biology approach allows transfer of an entire metabolic pathway from a medicinal plant to a biomass crop. ELife 5:e13664

Gisby MF, Mudd EA, Day A (2012) Growth of transplastomics cells expressing D-amino acid oxidase in chloroplasts is tolerant to D-alanine and inhibited by D-valine. Plant Physiol 160:2219-2226

Goldschmidt-Clermont M (1991) Transgenic expression of aminoglycoside adenyl transferase in the chloroplast: a selectable marker for site-directed transformation of Chlamydomonas. Nucleic Acids Res 19:4083-4089

Hager M, Biehler K, Illerhaus J, Ruf S, Bock R (1999) Targeted inactivation of the smallest plastid genome-encoded open reading frame reveals a novel and essential subunit of the cytochrome b6f complex. EMBO J 18:5834-5842

Hajdukiewicz PTJ, Allison LA, Maliga P (1997) The two RNA polymerases encoded by the nuclear and the plastid compartments transcribe distinct groups of genes in tobacco plastids. EMBO J 16:4041-4048

Huang F-C, Klaus SMJ, Herz S, Zou Z, Koop H-U, Golds TJ (2002) Efficient plastid transformation in tobacco using the aphA-6 gene and kanamycin selection. Mol Genet Genomics 268:19-27

Huang CY, Ayliffe MA, Timmis JN (2003) Direct measurement of the transfer rate of chloroplast DNA into the nucleus. Nature 422:72-76

Kuras R, Wollman F-A (1994) The assembly of cytochrome b6/f complexes: an approach using genetic transformation of the green alga Chlamydomonas reinhardtii. EMBO J 13:1019-1027

Kuroda H, Maliga P (2001) Complementarity of the 16 S rRNA penultimate stem with sequences downstream of the AUG destabilizes the plastid mRNAs. Nucleic Acids Res 29:970-975

Li W, Ruf S, Bock R (2011) Chloramphenicol acetyltransferase as selectable marker for plastid transformation. Plant Mol Biol 76:443-451
Lu Y, Rijzaani H, Karcher D, Ruf S, Bock R (2013) Efficient metabolic pathway engineering in transgenic tobacco and tomato plastids with synthetic multigene operons. Proc Natl Acad Sci USA 110:E623-E632

Lutz KA, Maliga P (2007) Construction of marker-free transplastomic plants. Curr Opin Biotechnol 18:107-114

Maliga P (2004) Plastid transformation in higher plants. Annu Rev Plant Biol 55:289-313

Mingeot-Leclercq M-P, Glupczynski Y, Tulkens PM (1999) Aminoglycosides: Activity and resistance. Antimicrob Agents Chemother 43:727-737

Monod C, Takahashi Y, Goldschmidt-Clermont M, Rochaix J-D (1994) The chloroplast ycf8 open reading frame encodes a photosystem II polypeptide which maintains photosynthetic activity under adverse growth conditions. EMBO J 13:2747-2754

Murashige T, Skoog F (1962) A revised medium for rapid growth and bio assays with tobacco tissue culture. Physiol Plant 15:473-497

Oey M, Lohse M, Kreikemeyer B, Bock R (2009a) Exhaustion of the chloroplast protein synthesis capacity by massive expression of a highly stable protein antibiotic. Plant J 57:436-445

Oey M, Lohse M, Scharff LB, Kreikemeyer B, Bock R (2009b) Plastid production of protein antibiotics against pneumonia via a new strategy for high-level expression of antimicrobial proteins. Proc Natl Acad Sci USA 106:6579-6584

Rogalski M, Ruf S, Bock R (2006) Tobacco plastid ribosomal protein $\mathrm{S} 18$ is essential for cell survival. Nucleic Acids Res 34:4537-4545

Ruf S, Kössel H, Bock R (1997) Targeted inactivation of a tobacco intron-containing open reading frame reveals a novel chloroplastencoded photosystem I-related gene. J Cell Biol 139:95-102

Ruf S, Hermann M, Berger IJ, Carrer H, Bock R (2001) Stable genetic transformation of tomato plastids and expression of a foreign protein in fruit. Nature Biotechnol 19:870-875

Ruf S, Karcher D, Bock R (2007) Determining the transgene containment level provided by chloroplast transformation. Proc Natl Acad Sci USA 104:6998-7002

Staub JM, Maliga P (1993) Accumulation of D1 polypeptide in tobacco plastids is regulated via the untranslated region of the psbA mRNA. EMBO J 12:601-606

Staub JM, Garcia B, Graves J, Hajdukiewicz PTJ, Hunter P, Nehra N, Paradkar V, Schlittler M, Carroll JA, Spatola L, Ward D, Ye G, Russell DA (2000) High yield production of a human therapeutic protein in tobacco chloroplasts. Nature Biotechnol 18:333-338

Stegemann S, Hartmann S, Ruf S, Bock R (2003) High-frequency gene transfer from the chloroplast genome to the nucleus. Proc Natl Acad Sci USA 100:8828-8833

Stegemann S, Keuthe M, Greiner S, Bock R (2012) Horizontal transfer of chloroplast genomes between plant species. Proc Natl Acad Sci USA 109:2434-2438

Svab Z, Maliga P (1991) Mutation proximal to the tRNA binding region of the Nicotiana plastid 16S rRNA confers resistance to spectinomycin. Mol Gen Genet 228:316-319

Svab Z, Maliga P (1993) High-frequency plastid transformation in tobacco by selection for a chimeric aadA gene. Proc Natl Acad Sci USA 90:913-917

Svab Z, Maliga P (2007) Exceptional transmission of plastids and mitochondria from the transplastomic pollen parent and its impact on transgene containment. Proc Natl Acad Sci USA 104:7003-7008

Svab Z, Hajdukiewicz P, Maliga P (1990) Stable transformation of plastids in higher plants. Proc Natl Acad Sci USA 87:8526-8530

Tenson T, Mankin A (2006) Antibiotics and the ribosome. Mol Microbiol 59:1664-1677

Tiller N, Bock R (2014) The translational apparatus of plastids and its role in plant development. Mol Plant 7:1105-1120 
Tregoning JS, Nixon P, Kuroda H, Svab Z, Clare S, Bowe F, Fairweather N, Ytterberg J, van Wijk KJ, Dougan G, Maliga P (2003) Expression of tetanus toxin fragment $C$ in tobacco chloroplasts. Nucleic Acids Res 31:1174-1179

Wurbs D, Ruf S, Bock R (2007) Contained metabolic engineering in tomatoes by expression of carotenoid biosynthesis genes from the plastid genome. Plant J 49:276-288

Ye G-N, Hajdukiewicz PTJ, Broyles D, Rodriguez D, Xu CW, Nehra N, Staub JM (2001) Plastid-expressed 5-enolpyruvylshikimate3 -phosphate synthase genes provide high level glyphosate tolerance in tobacco. Plant J 25:261-270

Ye G-N, Colburn SM, Xu CW, Hajdukiewicz PTJ, Staub JM (2003) Persistence of unselected transgenic DNA during a plastid transformation and segregation approach to herbicide resistance. Plant Physiol 133:402-410
Zhang J, Ruf S, Hasse C, Childs L, Scharff LB, Bock R (2012) Identification of cis-elements conferring high levels of gene expression in non-green plastids. Plant J 72:115-128

Zhang J, Khan SA, Hasse C, Ruf S, Heckel DG, Bock R (2015) Full crop protection from an insect pest by expression of long doublestranded RNAs in plastids. Science 347:991-994

Zhou F, Badillo-Corona JA, Karcher D, Gonzalez-Rabade N, Piepenburg K, Borchers A-MI, Maloney AP, Kavanagh TA, Gray JC, Bock R (2008) High-level expression of human immunodeficiency virus antigens from the tobacco and tomato plastid genomes. Plant Biotechnol J 6:897-913

Zou Z, Eibl C, Koop H-U (2003) The stem-loop region of the tobacco psbA 5' UTR is an important determinant of mRNA stability and translation efficiency. Mol Gen Genomics 269:340-349 